\title{
MATHEMATISCHE NACHRICHTEN
}

I M AUFTRAGE DER DEUTSCHEN AKADEMIE DER WISSENSCHAFTEN Z U BERLIN

HERAUSGEGEBEN VON

E R HAR D SCH M I T T

GEMEINSAM MIT

HEINRICH GRELL, GEORG HAMEL, HELMUT HASSE, H.L.SCHMID UND KURT SCHRÖDER

$\frac{\overline{10 . \mathrm{BAND}}}{1953}$

AK ADE M I E - VERLA G B B RLIN 


\section{Inhalt des 10. Bandes}

Banaschwwsk, Brknhakp, Uher den Sat\% von \%orn . . . . . . . . 181 186

Bavaschawsk, Bronhard, Üher die Konstruktion wohlgeordneter Mengen . 239-245

Brakket, Herbert, Eine Eigenschaft der klassischen Grenschen Funktionen erster und zweiter Art . . . . . . . . . . . . . . . . . . 5561

Dörgti, Kart, und Schuff, Hass Konrad, Über Jlimination in beliebigen Mergen mit allgemeinsten Operatioren . . . . . . . . . . . . . . . . . . 315 330

Wmbsrmax, Otтo, Das elektrostatische Selbstpotential äquidistanter Ladungen auf einer Kreislinic . . . . . . . . . . . . . . . . . . . . . 135 167

Joras, Haxs, Zwei Klassen von Flächen, deren Bestimmung von einem Integral der Telegraphengleichurg ahhăngt . . . . . . . . . . . . . . . . . . . 63 74

Joxas, Haxs, Die Differentialyleichung der Affinsphären in ciner neuen Gestalt $331 \ldots 352$

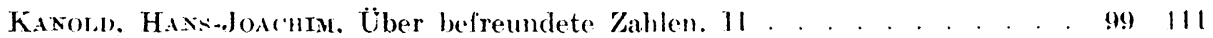

Kasch, Fnıркін, Zur Annäherung algebraischer Zahlen durch arithmetisch (harakterisierte rationale \%ahlen . . . . . . . . . . . . . . . . . . 85.98

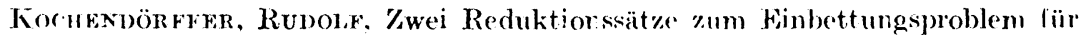
abclishe Algebren. . . . . . . . . . . . . . . . . . . . is st

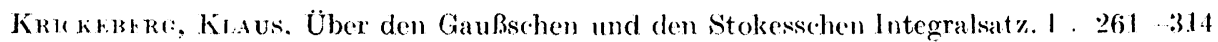

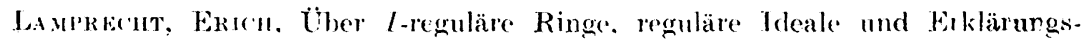
modulı. I . . . . . . . . . . . . . . 35:3 38:

Jabrak, Rebolf, /ar Topologie der Konfiguration von Desargues. 11 . . 179180

Mонк, Вкхsт, Integration von gewöhnlichen Differentialgleichungen mit konstanten Kocffizienten mittels Operatorenrechrung . . . . . . . . . 149

Monr, Exsst, Uber die Funktionalgleichurg des arithmetisch-geometrischen Mittels. . . . . . . . . . . . . . . . . . . . . 129 1333

Monr, Ersst, Einfacher Beweis des verallgemeinerten Determirantensitzes ron Sylvester nebst einer Verschärfung . . . . . . . . . . . . . . . 257 -260 
Practar, Kart, Über Zahlen der Form $a^{2}+b^{2}$ in einer arithmetischen Progression

Schmeidler, Werner, Algebraische Integralgleichungen. II

Schmtor, Jürgex, Beiträge zur Filtertheorie. II . . . . . . . . . . . . . $197 \quad 232$

Schröder, JoHaxx, Fehlerabschätzungen zur Störungsrechnung bei linearen Eigenwertproblemen mit Operatoren eines Hilbertschen Raumes. . . . 113-128

Schuff, Hans Konrad, siehe Dörge, KarL

Wolf, Pacl, Galoissche Algebren mit vorgegebener Galoisgruppe über einem Teilkörper des Grundkörpers. II

ZaACharias, MaX, Über eine mit der Bydžovskýschen Konfiguration $\left(12_{4}, 16_{3}\right)$ verbundene Hessesche Konfiguration . . . . . . . . . . . . . . . . . 187-196

Zeller, Kart, Transformationen des Durchschnitts und der Vereinigung von Folgenräumen . . . . . . . . . . . . . . . . . . . . 175-177

Zlámat, Mrloš, Asymptotische Eigenschaften der Lösungen linearer Differentialgleichungen . . . . . . . . . . . . . . . . . . . . . . . . . . . . . . . . . . . 169 174

Berichtigungen . . . . . . . . . . . . . . . . 383 


\title{
Zur Annäherung algebraischer Zahlen durch arithmetisch charakterisierte rationale Zahlen
}

\author{
Von Friedrich Kasch in Göttingen
}

(Eingegangen am 5.1.1953)

Ergebnisse

Von C. L. Siegel stammt die Idee, bei der Annäherung algebraischer Zahlen durch rationale die arithmetischen Eigenschaften der Näherungszahlen zu berücksichtigen ([1], S. 736, Fußn.). In anderer Weise wurde diese Idee von Th. Schneider herangezogen ([3]) und von K. MAHLER weiter ausgebaut ([1], [2]). Schließlich bewies Th. Schneider kürzlich einen Satz, der seine und die Mahlerschen Ergebnisse zusammenfaßt und verallgemeinert ([4]). Dieser Satz kann folgendermaßen formuliert werden:

Es sei $\frac{p_{1}}{q_{1}}, \frac{p_{2}}{q_{2}}, \frac{p_{3}}{q_{3}}, \ldots$ eine Folge verschiedener rationaler Zahlen mit folgenden Eigenschaften:

1. $q_{i} \leqq q_{i+1},\left(p_{i}, q_{i}\right)=1 \quad(i=1,2,3, \ldots)$;

2. Die Z̈ahlen $p_{i}$ und $q_{i}(i=1,2,3, \ldots)$ besitzen Faktorzerlegungen in ganze Zahlen:

$$
p_{i}=p_{i}^{\prime} p_{i}^{\prime \prime}, \quad q_{i}=q_{i}^{\prime} q_{i}^{\prime \prime},
$$

wobei die $p_{i}^{\prime \prime}$ und $q_{i}^{\prime \prime}$ Potenzprodukte von nur endlich vielen, von $i$ unabhängigen Primzahlen sind. Es werden bezeichnet:

$$
\varlimsup_{i \rightarrow \infty} \frac{\log p_{i}^{\prime}}{\log p_{i}}=\omega, \quad \varlimsup_{i \rightarrow \infty} \frac{\log q_{i}^{\prime}}{\log q_{i}}=\sigma ;
$$

3. Die Zahlen $\frac{p_{i}}{q_{i}}(i=1,2,3, \ldots)$ sind Lösungen der Ungleichung

$$
\left|\alpha-\frac{p_{i}}{q_{i}}\right|<q_{i}^{-\prime \prime}
$$

wobei a eine reelle, irrationale algebraische Zahl und $\mu>\omega+\sigma$ ist. Dann gilt

$$
\varlimsup_{i \rightarrow \infty} \frac{\log q_{i+1}}{\log q_{i}}=\infty
$$

Dieser Satz, der ohne die arithmetischen Voraussetzungen für $\mu=2$ bereits falsch ist ([5]), erlaubt im Spezialfall Exponentenwerte $\mu>0$. Diese Tatsache ist insbesondere für die Anwendung zur Konstruktion transzendenter Zahlen von Interesse. Th. Schneider stellte in diesem Zusammenhang die Frage, ob es gelingt, eine ähnliche Aussage zu beweisen, wenn etwa die Näherungsnenner 
„,im wesentlichen“ aus Fakultäten bestehen. Daß dies möglich ist, soll hier gezeigt werden.

Dabei wird zugleich eine Verschärfung der Behauptung

$$
\varlimsup_{i \rightarrow \infty} \frac{\log q_{i+1}}{\log q_{i}}=\infty
$$

hergeleitet, die eine gewisse Aussage darüber macht, daß „große" Quotienten $\frac{\log q_{i+1}}{\log q_{i}}$ in der Folge aller dieser Quotienten nicht ,weit" auseinanderliegen. Diese Verschärfung ergibt sich bereits aus der Beweismethode des vorstehenden Satzes und hat auch für diesen Gültigkeit.

Wir zeigen:

Satz 1. Es sei $\frac{p_{1}}{q_{1}}=\zeta_{1}, \frac{p_{2}}{q_{2}}=\zeta_{2}, \frac{p_{3}}{q_{3}}=\zeta_{3}, \ldots$ eine Folge verschiedener rationaler Zahlen mit folgenden Eigenschaften:

1. $q_{i} \leqq q_{i+1},\left(p_{i}, q_{i}\right)=1 \quad(i=1,2,3, \ldots)$;

2. Die $q_{i}$ besitzen eine Faktorzerlegung in ganze Zahlen $q_{i}=q_{i}^{\prime} q_{i}^{\prime \prime}$, und es sei

$$
\varlimsup_{i \rightarrow \infty} \frac{\log q_{i}^{\prime}}{\log q_{i}}=\omega_{1}
$$

3. Es gibt ganze rationale Zahlen $f_{i}$ mit $q_{i}^{\prime \prime} f_{i}=l_{i}$ !, und es sei

$$
\varlimsup_{i \rightarrow \infty} \frac{\log f_{i}}{\log q_{i}}=\omega_{2} ;
$$

4. Die Zahlen $\frac{p_{i}}{q_{i}}$ genügen der Ungleichung

$$
\left|\alpha-\frac{p_{i}}{q_{i}}\right|<q_{i}^{-\mu}
$$

wobei a eine reelle algebraische Zahl und

ist.

$$
\mu>1+\omega_{1}+\omega_{2}\left(\left(1-\omega_{1}\right)\left(2+\omega_{2}\right)+\omega_{1}^{2}\right)
$$

Dann ist

$$
\varlimsup_{i \rightarrow \infty} \frac{\log q_{i+1}}{\log q_{i}}=\infty .
$$

Darüber hinaus gilt: Für jede Teilfolge der gegebenen Folge mit der Bezeichnung

$$
\zeta_{a_{1}, 1}, \zeta_{a_{1}, 1}, \ldots, \zeta_{a_{1}, b_{1}}, \zeta_{a_{2}, 1}, \ldots, \zeta_{a_{2}, b_{2}}, \zeta_{a_{2}, 11}, \ldots
$$

und

für die gilt:

$$
\lim _{\substack{i \rightarrow \infty \\ 0 \leqq n<b_{i}}} \frac{\log q_{a_{i}, n+1}}{\log q_{a_{i}, n}}=\eta,
$$

$$
\begin{gathered}
\lim _{i \rightarrow \infty} \frac{\log b_{i}}{\log q_{a_{i}, 0}}=\infty, \text { falls } \eta=1 . \\
\lim _{i \rightarrow \infty} b_{i}=\infty, \quad \text { falls } \eta>1 .
\end{gathered}
$$

ist

$$
\varlimsup_{\substack{i \rightarrow \infty \\ 0 \leqq n<b_{i}}} \frac{\log q_{a_{i}, n+1}}{\log q_{a_{i}, n}}=\infty .
$$


Daß (2) aus der letzten Behauptung folgt, ist sofort einzusehen. Dazu hat man nur die ursprüngliche Folge so zu unterteilen, daß $\varlimsup_{i \rightarrow \infty} \frac{\log b_{i}}{\log q_{a_{i}, 0}}=\infty$ gilt,
was offenbar möglich ist.

Aus dem Beweis ergibt sich, daß man diesen Satz auch mit dem zuvor angegebenen Satz kombinieren und etwa über die $p_{i}$ und $q_{i}^{\prime}$ noch die dort für die $p_{i}$ und $q_{i}$ angegebenen Voraussetzungen machen könnte, um damit $\mu$ weiter zu verkleinern. Darauf soll jedoch hier nicht eingegangen werden, ebensowenig auf eine mögliche Verallgemeinerung beider Sätze auf die Annäherung algebraischer Zahlen durch algebraische Zahlen, die für den Fall, daß über die Näherungswerte keine arithmetischen Voraussetzungen gemacht werden, bereits beliannt ist ([3]).

Satz 1 kann zur Konstruktion transzendenter Zahlen benutzt werden. Wir beweisen dazu den folgenden Satz.

Satz 2. Die Reihe

$$
\xi=\sum_{\nu=0}^{\infty} \varrho_{\nu}=\sum_{\nu=0}^{\infty} \frac{r_{\nu}}{u_{\nu} !}
$$

ist transzendent, wenn folgende Voraussetzungen erfüllt sind:

1. Die $u_{\nu}$ sind natürliche Zahlen mit $\lim _{\nu \rightarrow \infty} \frac{u_{v+1}}{u_{\nu}}=c>1$;

2. $r_{v}=\frac{\varepsilon_{v}}{t_{v}}$, wobei $s_{v}, t_{v}$ ganze rationale Zahlen sind, die den Bedingungen $0<t_{v}$ und $\left|s_{v^{v}}\right|, t_{v} \leqq B^{d^{v}}$ genügen, wobei $B>0$ eine beliebige Konstante und $d<c$ ist;

3. Es gibt eine unendliche Teilfolge der Folge aller Reihenglieder, deren Elemente von 0 verschieden sind und für die mit der Bezeichnung

$$
\varrho_{a_{1}, 0}, \varrho_{a_{1}, 1}, \ldots, \varrho_{a_{1}, b_{1}}, \varrho_{a_{2}, 0}, \ldots, \varrho_{a_{2}, b_{2}}, \varrho_{a_{3}, 0}, \ldots
$$

gilt:

$$
\begin{aligned}
& \text { a) } \lim _{i \rightarrow \infty} b_{i}=\infty, \\
& \text { b) } \varlimsup_{i \rightarrow \infty} \frac{u_{a_{i}, n+1}}{u_{a_{i}, n}}<\infty .
\end{aligned}
$$

In diesem Satz ist zum Beispiel die Aussage enthalten, daß bei sonst gleichen Voraussetzungen die Potenzreihe

$$
\xi(v)=\sum_{\nu=0}^{\infty} \varrho_{\nu} x^{v}
$$

für rationale Argumente $\neq 0$ transzendente Funktionswerte besitzt.

Die Voraussetzungen 1. und 3. des Satzes sind etwa erfüllt, wenn $u_{v}=\left[c^{v}\right]$ mit beliebigem $c>1$ und $r_{v} \neq 0$ gesetzt wird.

Zum Beweis von Satz 2 wird von Satz 1 sowohl die anfangs erwähnte Verschärfung von (2) als auch die Verkleinerung des Exponenten $\mu$ bis auf $\mu>1$ für $\omega_{1}=\omega_{2}=0$ tatsächlich gebraucht, so daß Satz 2 nicht aus den bisher bekannten Approximationssätzen folgen dürfte.

Beim Beweis beider Sätze wird von gewissen arithmetischen Eigenschaften der Fakultäten Gebrauch gemacht, die in den Hilfssätzen 4 und 5 hergeleitet werden. 


\section{Beweis von Satz 1}

1. Der Beweis schließt sich an den des anfangs erwähnten Satzes an und benutzt die dort angegebenen Hilfssätze, die ohne Beweis übernommen werden ${ }^{1}$ ).

Hilfssatz 1. $r_{1}, r_{2}, \ldots, r_{k}$ seien natürliche Zahlen. Man schneide den Quader

$$
-\frac{1}{2} \leqq \frac{x_{x}}{r_{*}} \leqq \frac{1}{2} \quad(i=1, \ldots, n)
$$

mit der $(k-1)$-dimensionalen Ebene $\sum_{x=1}^{k} \frac{x_{x}}{r_{x}}=t$. Der Quotient $Q$ aus der Anzahl der Gitterpunkte, die in dem kleineren Quaderabschnitt (mit Einschluß seiner Oberfläche) liegen, und der Anzahl der Gitterpunkte des ganzen Quaders ist für $t=\varepsilon k(\varepsilon>0$, unabhängig von $k)$ und hinreichend großes $k$ beliebig klein.

Zusatz. Daraus folgt sofort, daß auch der Quotient $Q^{\prime}$ aus der Anzahl der Gitterpunkte des Quaderabschnitts

$$
-\frac{1}{2} \leqq \frac{x_{x}}{r_{x}} \leqq \frac{1}{2} \quad(\varkappa=1, \ldots, k), \quad \sum_{x=1}^{k} \frac{x_{x}}{r_{\varkappa}} \geqq t
$$

und der Anzahl der Gitterpunkte des Quaderabschnitts

$$
-\frac{1}{2} \leqq \frac{x_{x}}{r_{x}} \leqq \frac{1}{2} \quad(\varkappa=1, \ldots, k), \quad\left|\sum_{x=1}^{k} \frac{x_{\varkappa}}{r_{x}}\right|<t
$$

für $t=\varepsilon k$ und hinreichend großes $k$ beliebig klein ist.

Hilfssatz 2. Es sei $\alpha \neq 0$ eine reelle algebraische Zahl vom Grade $n \geqq 2$. Ferner sei $r=\max \left(r_{1}, \ldots, r_{k}\right)$. Setzt man $t=\varepsilon k \quad(\varepsilon>0$, unabhängig von $k)$ und wählt $k$ derart, daß Hilfssatz 1 mit $Q^{\prime}<\frac{1}{2 n}$ erfüllt ist, so gilt: Es gibt ein nicht identisch verschwindendes Polynom $R\left(z_{1}, \ldots, z_{k}\right)$ mit ganzrationalen Koeffizienten und höchstens vom Grade $r_{\varkappa}$ in $z_{\varkappa}(\varkappa=1, \ldots, k)$ so, da $\beta$

1. die Werte

$$
\left.\frac{\partial \tau_{1}+\cdots+\tau_{k} R\left(z_{1}, \ldots, z_{k}\right)}{\partial z_{1}^{\tau_{1}} \cdots \partial z_{k}^{\tau_{k}}}\right|_{z_{1}=\cdots=z_{k}=0}=\left.\frac{\partial^{\tau_{1}+\cdots+\tau_{k} R\left(z_{1}, \ldots, z_{k}\right)}}{\partial z_{1}^{\tau_{1}} \cdots \partial z_{k}^{\tau_{k}}}\right|_{z_{1}=\cdots=z_{k}=\alpha}=0
$$

sind, falls

$$
\sum_{*=1}^{k} \frac{\tau_{*}}{r_{*}} \leqq k\left(\frac{1}{2}-\varepsilon\right) \quad \text { und } \quad \sum_{*=1}^{k} \frac{\tau_{*}}{r_{x}} \geqq k\left(\frac{1}{2}+\varepsilon\right)
$$

ist und $\tau_{1}, \ldots, \tau_{k}$ nichtnegative ganzrationale Zahlen bedeuten;

2. die Ungleichung

$$
\left|\frac{\partial^{\varrho_{1}}+\cdots+\varrho_{k} R\left(z_{1}, \ldots, z_{k}\right)}{\varrho_{1} ! \cdots \varrho_{k} ! \partial z_{1}^{\varrho_{1}} \cdots \partial z_{\tau}^{\varrho_{k}}}\right|<\gamma_{1}^{r} \prod_{\varkappa=1}^{k}\left(1+\left|z_{\varkappa}\right|\right)^{r_{\varkappa}}
$$

1) Der Beweis könnte auch nach dem Mahlerschen Vorbild [1] geführt werden, wobei man insbesondere ohne den Schubfachschluß auskommt. Wir benutzen die Schneidersche Methode, da wir dann aus [4] außer den Hilfssätzen weitere Ergebnisse unmittelbar übernehmen können. 
erfüllt ist, wobei die $\varrho_{x} \geqq 0(*=1, \ldots, k)$ ganzrationale Zahlen sind and die natürliche Zahl $\gamma_{1}$ nur von $k, \varepsilon, \alpha$ abhängt ${ }^{2}$ ).

Hilfssatz 3 . Es seien $p_{1}, \ldots, p_{k}, q_{1}, \ldots, q_{k}$ ganzrationale Zahlen mit $q_{\varkappa}>0$ und $\left(p_{\varkappa}, q_{\varkappa}\right)=1(\varkappa=1, \ldots, k)$. Ferner sei $R\left(z_{1}, \ldots, z_{k}\right)$ das in Hilfs. satz 2 bestimmte Polynom. Dann gilt: Ist

$$
\log q_{\varkappa}>\gamma_{2}{ }^{r} \prod_{\lambda=\kappa+1}^{k}\left(r_{\lambda}+1\right)^{2} \quad(\varkappa=1, \ldots, k)
$$

$\left(\right.$ für $\varkappa=k$ bedeute $\prod_{i=r+1}^{k}\left(r_{i}+1\right)^{2}$ die Zahl 1$)$, so existieren $k-1$ Zahlen

so, daß

$$
\varrho_{*}<\prod_{\lambda=x+1}^{k}\left(r_{\lambda}+1\right) \quad(\varkappa=1, \ldots k-1)
$$

$$
\left.\frac{\partial^{\varrho_{1}}+\cdots+\varrho_{k-1} R\left(z_{1}, \ldots, z_{k}\right)}{\partial z_{1}^{\varrho_{1}} \ldots \partial z_{k-1}^{\varrho_{k}-1}}\right|_{z_{1}=\frac{p_{1}}{q_{1}}, \ldots, z_{k}=\frac{p_{k}}{q_{k}}} \neq 0
$$

ist. Selbstverständlich folgt dann $\varrho_{x} \leqq r_{*}$.

2. Wir stellen zunächst fest, daß man ohne Einschränkung statt (1) auch

$$
\lim _{i \rightarrow \infty} \frac{\log q_{i}^{\prime}}{\log q_{i}}=\omega_{1}
$$

voraussetzen kann. Wegen $l_{i} ! \leqq q_{i}^{2}+\omega_{2}$ für fast alle $i$ und auf Grund der Stirlingschen Formel gilt $l_{i} \leqq \log q_{i}$ für fast alle $i$. Demnach kann man von der angegebenen Zerlegung $q_{i}=q_{i}^{\prime} q_{i}^{\prime \prime}$ zu einer Zerlegung $q_{i}=\bar{q}_{i}^{\prime} \bar{q}_{i}^{\prime \prime} \operatorname{mit} \lim _{i \rightarrow \infty} \frac{\log \bar{q}_{i}^{\prime}}{\log q_{i}}=\omega_{1}$ und $\bar{q}_{i}^{\prime \prime} \bar{f}_{i}=\bar{l}_{i}$ ! übergehen, wobei $\bar{f}_{i} \leqq f_{i}$ und $\bar{l}_{i} \leqq l_{i}$ gilt. Dann ist $\overline{\lim }_{i \rightarrow \infty} \frac{\log \bar{f}_{i}}{\log q_{i}}=\bar{\omega}_{2} \leqq \omega_{2}$. Gilt der Satz für $\omega_{1}$ und $\bar{\omega}_{2}$, so auch für $\omega_{1}$ und $\omega_{2}$.

Ferner bemerken wir, daß $q_{i}<q_{i+1}$ für fast alle $i$ gilt, so daß für jede wachsende Folge von Indizes die Folge der zugehörigen $q_{i}$ gegen $\infty$ strebt.

3. Der Beweis wird indirekt geführt. Wir nehmen an, daß es eine Folge (3) gibt, für die

$$
\varlimsup_{\substack{i \rightarrow \infty \\ 0 \leqq n<b_{i}}} \frac{\log q_{a_{i}, n+1}}{\log q_{a_{i}, n}}=\sigma^{\prime}<\infty
$$

ist. Aus dieser Annahme kann zunächst gefolgert werden, daß es dann zu zwei beliebigen festen Zahlen $B, C$ mit $0<B<C$ eine unendliche Folge von natürlichen Zahlen $u_{1}, u_{2}, \ldots$ gibt und zu jedem $u_{i}$ eine Zahl $a_{j}$, bezeichnet mit $a_{j}=a_{(i)}$, so daß mit $b_{j}=b_{(i)}$ gilt:

$$
\frac{\log q_{a_{(i)}, 0}}{B}<u_{i}<\frac{\log q_{a_{(i)}, b_{(i)}}}{C} .
$$

Um dies einzusehen, ordne man jeder Zahl $a_{j}$ die ganze Zahl $u_{i}$ zu, die der Ungleichung

$$
\frac{\log q_{a_{j}, 0}}{u_{i}}<B \leqq \frac{\log q_{a_{j}, 0}}{u_{i}-1}
$$

2) Ebenso sollen alle noch auftretenden Zahlen $\gamma_{2}, \gamma_{3}, \gamma_{4}, \ldots$ nur von $k, \varepsilon, \alpha$ und einer noch einzuführenden $Z$ ahl $\delta$, nicht aber von $r_{1}, \ldots, r_{k}$ abhängen. 
genügt. Dann ist

Ist

$$
\frac{\log q_{a_{j}, 0}}{B}<u_{i} \leqq \frac{1}{B} \frac{u_{i}}{u_{i}-1} \log q_{a_{j}, 0}<\frac{2}{B} \log q_{a_{j}, 0} .
$$

$$
\frac{2}{B} \log q_{a_{j}, 0}<\frac{1}{C} \log q_{a_{j}, b_{j}}
$$

erfüllt, so gilt (10). Ist $\eta=1$, so folgt aus $q_{i}<q_{i+1}$ und (5)

$$
q_{a_{j}, b_{j}}>q_{a_{j}, 0}+b_{j} \geqq q_{a_{j}, 0}+q_{a_{i}, 0}^{N}
$$

mit beliebig vorgegebenem $N$ für hinreichend großes $j$. Folglich gilt (11) mit $N>\frac{2 C}{B}$. Ist $\eta>1$, so folgt aus (4) mit $\eta-1>\delta>0$

$$
q_{a_{j}, b_{j}} \geqq q_{a_{j}, 0}^{(\eta-\delta)^{b_{j}}} \text {. }
$$

Wegen $\lim _{i \rightarrow \infty} b_{i}=\infty$ und $\eta-\delta>1$ ist dann für hinreichend großes $j$

$$
\frac{2}{B} \log q_{a_{j}, 0} \leqq \frac{(\eta-\delta)^{b_{j}}}{C} \log q_{a_{j}, 0} ;
$$

also gilt auch jetzt (11). Da mit $j \rightarrow \infty$ auch die zugeordneten $u_{i}$ gegen $\infty$ wachsen, gibt es darunter eine unendliche Folge $u_{1}<u_{2}<\cdots$ der behaupteten Art.

Es seien im folgenden $k, r_{1}, \ldots, r_{k}$ die in den Hilfssätzen auftretenden natürlichen Zahlen, über die noch genauer verfügt wird. Wir setzen

$$
B=r_{k}^{-3^{k-1}}, \quad C=\left(\sigma^{\prime}+1\right) r_{k}=\sigma r_{k}
$$

und verstehen unter $u_{1}, u_{2}, \ldots$ und $a_{(1)}, a_{(2)}, \ldots$ die zugehörigen Folgen, die (10) genügen. Dann wird behauptet: $Z u$ fast allen $u_{i}$ dieser Folge und jeder der Zahlen $d_{\varkappa}=k \cdots \varkappa$ mit $\varkappa=1, \ldots, k$ gibt es ein $q_{a_{(i)}, n}$ mit $0 \leqq n<b_{(i)}$, für das

$$
u_{i} r_{k}^{-s^{d} \%}<\log q_{a_{(i)}, n}<\sigma u_{i} r_{k}^{-3^{d *} \%}
$$

erfüllt ist. Auf Grund von (10) gilt zunächst

Ferner ist

$$
\log q_{a_{(i)}, 0}<u_{i} r_{k}^{-3^{k-1}}, \quad \sigma u_{i} r_{k}<\log q_{a_{(i)}, b_{(i)}} .
$$

$$
\log q_{a_{(i)}, n+1}<\sigma \log q_{a_{(i)}, n} \quad\left(0 \leqq n<b_{(i)}\right) .
$$

Aus beiden Beziehungen zusammen folgt aber (12).

$\mathrm{Zu}$ jedem $u_{i}$ von einem festen Index an und jedem $d_{\varkappa}(\varkappa=1, \ldots, k)$ denke man sich nun ein $q_{a_{(i)}, n}$, welches (12) genügt, ausgezeichnet. Für diese ausgezeichneten $q_{a_{(i)}, n}$ kann man die Schreibweise $q_{a_{(i)}, n}=q\left(u_{i}, d_{*}\right)$ einführen. Dann folgt aus (12)

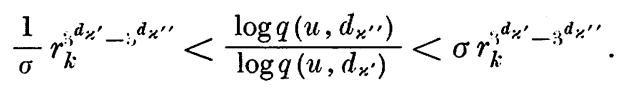

Aus der Folge der $u_{i}$ wird nun eine solche unendliche Teilfolge ausgewählt, daß die $k$ Grenzwerte

$$
\lim \left[r_{k} \frac{\log q\left(u_{i}, d_{k}\right)}{\log q\left(u_{i}, d_{x}\right)}\right]=r_{\varkappa} \quad(\varkappa=1, \ldots, k)
$$


für die $u_{i}$ aus dieser Teilfolge existieren. Das ist möglich, denn bei festem $r_{k}$ und veränderlichem $u_{i}$ liegen die Ausdrücke $r_{k} \frac{\log q\left(u_{i}, d_{k}\right)}{\log q\left(u_{i}, d_{\varkappa}\right)}$ wegen (13) in einem endlichen Intervall.

Bei den weiteren Überlegungen wird die so gewonnene Teilfolge zugrunde gelegt, die wir der einfachen Schreibweise wegen wieder $u_{1}, u_{2}, \ldots$ nennen.

Das zu jedem $u_{i}$ vermöge (12) gehörende $k$-tupel ron Näherungsnennern werde mit

$$
q\left(u_{i}, d_{1}\right)=q_{1}, \ldots, q\left(u_{i}, d_{k}\right)=q_{k}
$$

bezeichnet. Dann erfüllen dieses $k$-tupel für fast alle $u_{i}$ die Voraussetzung (8) von Hilfssatz 3 .

Auf der rechten Seite der Ungleichung (8) steht nämlich ein von $u_{i}$ unabhängiger Ausdruck, während die $q_{\varkappa}$ mit $u_{i}$ wachsen. $Z$ u jedem $k$-tupel von zugehörigen Näherungswerten $\frac{p_{*}}{q_{*}}=\zeta_{*}(\varkappa=1, \ldots, k)$ gibt es dann nichtnegative ganze Zahlen

so daß

$$
\varrho_{*}<\prod_{i=x+1}^{k}\left(r_{i}+1\right) \quad(\varkappa=1, \ldots, k-1),
$$

$$
\left.\frac{\partial^{\varrho_{1}}+\cdots+\varrho_{k-1} R\left(z_{1}, \ldots, z_{k}\right)}{\varrho_{1} ! \cdots \varrho_{k-1} ! \partial z_{1}^{\varrho_{1}} \cdots \partial z_{k-1}^{\varrho_{k-1}}}\right|_{z_{1}=\zeta_{1}, \ldots, z_{k}=\zeta_{k}} \neq 0
$$

ist. Für diesen Ausdruck führen wir die abkürzende Schreibweise $R_{(\varrho)}((\zeta))$ ein.

4. Es soll nun ein möglichst kleiner ganzrationaler Faktor $Q>0$ so bestimmt werden, daß $Q R_{(\underline{o})}((\zeta))$ ganzrational, also

ist.

$$
\left|Q R_{(\varrho)}((\zeta))\right| \geqq 1
$$

Um dabei die arithmetischen Voraussetzungen über die Näherungsnenner heranziehen zu können, zeigen wir zunächst:

Hilfssatz 4. Sei mit natürlichen Zahlen $m, s, t$

$$
(s !)^{m} \leqq t ! ;
$$

dann gibt es einen ganzrationalen Faktor $g=g(s, m, t)$ mit

$$
(s !)^{m} \mid t ! g,
$$

der bei festem $m$ und gleichmäßig in $t$

gen.ügt.

$$
\lim _{s \rightarrow \infty} \frac{\log g}{\log (s !)}=0
$$

Beweis. Offenbar gilt

$$
(s !)^{m} \mid(m s) ! .
$$

Ist $m s \leqq t$, so gilt die Behauptung mit $g=1$. Ist $t<m s$, so ist mit $g=\frac{(m s) \text { ! }}{t !}$ die erste Bedingung erfüllt. Auf Grund der Voraussetzung ist

$$
\frac{(m s) !}{t !} \leqq \frac{(m s) !}{(s !)^{m}}
$$

und durch Anwendung der Stirlingschen Formel folgt hieraus mit einer geeigneten Konstanten $v=v(m)$

$$
\frac{(m s) !}{(s !)^{m}} \leqq v^{s}
$$


Da andrerseits $s ! \geqq s^{s(1-\delta)}$ mit $1>\delta>0$ für fast alle $s$ ist, gilt wie behauptet bei festem $m$ und gleichmäßig in $t$

$$
\lim _{s \rightarrow \infty} \frac{\log g}{\log (s !)} \leqq \lim _{s \rightarrow \infty} \frac{s \log v}{(1-\delta) s \log s}=0 .
$$

Auf Grund dieses Hilfssatzes können wir zur Bestimmung von $Q$ die arithmetischen Voraussetzungen heranziehen.

Da für $\omega_{1}=1$ die Behauptung des Satzes bereits in der des anfangs erwähnten Satzes enthalten ist, können wir der einfachen Abschätzung halber im folgenden $\omega_{1}<1$, also $1-\omega_{1}>0$ voraussetzen.

Zunächst folgt aus (14) zu vorgegebenem $\delta$ mit $1-\omega_{1}>\delta>0$ und für hinreichend großes $u_{i}$

also auf Grund von (1)

$$
q_{\varkappa}^{\frac{r_{\varkappa}}{r_{k}}} \leqq q_{k}
$$

$$
q_{\varkappa}^{\prime \prime \prime}{ }^{\left(1-\omega_{1}-\delta\right)} \frac{r_{\varkappa}}{r_{k}} \leqq q_{k}^{\prime \prime}{ }^{\left(1-\omega_{1}+\delta\right)}
$$

ferner ist

$$
f_{\chi} \leqq q_{\%}^{\omega_{2}+\delta} \leqq q_{\varkappa}^{\prime \prime}{ }^{\left(\omega_{2}+\delta\right)\left(1-\omega_{1}+\delta\right)} \leqq q_{k}^{\prime \prime}{ }^{\left(\omega_{2}+\delta\right) \frac{\left(1-\omega_{1}+\delta\right)^{2}}{1-\omega_{1}-\delta}} \frac{r_{k}}{r_{\varkappa}}
$$

und folglich

mit

$$
\left(q_{x}^{\prime \prime} f_{x}\right)^{\frac{r_{x}}{r_{k}}} \leqq\left(q_{k}^{\prime \prime} f_{k}\right)^{\beta}
$$

$$
\beta=\frac{1-\omega_{1}+\delta}{1-\omega_{1}-\delta}\left(1+\left(\omega_{2}+\delta\right)\left(1-\omega_{1}+\delta\right)\right) .
$$

Also gilt

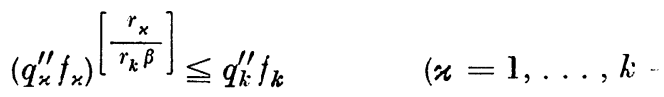

und auf Grund von Hilfssatz 4 folgt daraus

mit

$$
\left(q_{\varkappa}^{\prime \prime} f_{\varkappa}\right)^{\left[\frac{r_{\varkappa}}{r_{k} \beta}\right]} \mid q_{k}^{\prime \prime} f_{k} g_{\varkappa}
$$

$$
\lim _{u_{i} \rightarrow \infty} \frac{\log g_{x}}{\log \left(q_{x}^{\prime \prime} f_{x}\right)}=0
$$

Dann gilt

mit

$$
q_{*}^{\tau_{*}} \mid\left(q_{k} f_{k} g_{*}\right)^{\beta_{*}^{\prime}}
$$

$$
\beta_{*}^{\prime}=\left[\frac{\tau_{\varkappa}}{\left[\frac{r_{\varkappa}}{r_{k} \beta}\right]}+1\right] \leqq\left[\frac{\tau_{*} r_{k} \beta+r_{\varkappa}-r_{k} \beta}{r_{\varkappa}-r_{k} \beta}\right]=\beta_{\varkappa}
$$

für $x=1, \ldots, k-1$ und $\beta_{k}=\tau_{k}$. Folglich gilt dann auch

$$
\prod_{x=1}^{k} q_{\varkappa}^{\prime \prime \tau_{\kappa}} \mid\left(q_{k}^{\prime \prime} f_{k}\right)^{\sum_{x=1}^{k} \beta_{*}} \prod_{x=1}^{k} g_{\varkappa}^{\beta_{*}}
$$


mit

$$
\sum_{*=1}^{k} \beta_{\varkappa} \leqq\left[r_{k} \beta \sum_{\varkappa=1}^{k-1} \frac{\tau_{*}}{r_{\varkappa}-r_{k} \beta}+\frac{\tau_{k}}{r_{k}}+k\right] .
$$

Um diesen Ausdruck weiter abzuschätzen, zeigen wir, daß $\sqrt{r_{\kappa}}>r_{k}$ $(\varkappa=1, \ldots, k-1)$ ist, also für die Nenner in der Summe von (16)

$$
r_{\varkappa}-r_{k} \beta>(1-\delta) r_{\varkappa}
$$

für hinreichend großes $r_{k}$ gilt. Aus (13) und (14) folgt

$$
\frac{1}{\sigma} r_{k}^{3^{d_{\varkappa}}-3^{d_{k}}+1}-1<r_{\varkappa}<\sigma r_{k}^{3^{d_{\varkappa}}-3^{d_{k}}+1}
$$

Da außerdem offenbar die Ungleichung

$$
3^{d_{x}}-3^{d_{k}}+1>3\left(3^{d_{*+1}}-3^{d_{k}}+1\right)
$$

besteht, ergibt sich

$$
r_{\varkappa}>\frac{1}{\sigma} r_{k}^{3\left(3^{d_{x+1}}-3^{d_{k}}+1\right)}-1>\frac{1}{\sigma}\left(\frac{r_{x+1}}{\sigma}\right)^{3}-1 .
$$

Für hinreichend großes $r_{k}$ folgt dann

$$
r_{\varkappa}>\left(r_{\varkappa+1}+1\right)^{2}
$$

also wie behauptet $\sqrt{r_{\varkappa}}>r_{k}$ für $\varkappa=1, \ldots, k-1$. Damit wird aus (16)

$$
\sum_{*=1}^{k} \beta_{\varkappa} \leqq\left[k+\frac{r_{k} \beta}{1-\delta} \sum_{*=1}^{k} \frac{\tau_{\varkappa}}{r_{\varkappa}}\right] \leqq\left[k+\frac{r_{k} \beta}{1-\delta} k\left(\frac{1}{2}+\varepsilon\right)\right],
$$

wobei die letzte Ungleichung gilt, weil auf Grund von Hilfssatz 1 für alle in $R\left(z_{1}, \ldots, z_{k}\right)$ und damit erst recht für alle in $\frac{\partial^{\varrho_{1}+\cdots+\varrho_{k-1}} R\left(z_{1}, \ldots, z_{k}\right)}{\varrho_{1} ! \cdots \varrho_{k-1} ! \partial z_{1}^{\varrho_{1}} \cdots \partial z_{k-1}^{e_{k-1}}}$ tatsächlich auftretenden Potenzprodukte $z_{1}^{\tau_{1}} \ldots z_{k}^{\tau_{k}}$

$$
\sum_{x=1}^{k} \frac{\tau_{*}}{r_{x}} \leqq k\left(\frac{1}{2}+\varepsilon\right)
$$

ist. Da wegen (18) offenbar $\beta_{\varkappa} \leqq r_{\varkappa}(\varkappa=1, \ldots, k)$ ist, gilt ferner

Damit haben wir in

$$
\prod_{x=1}^{k} g_{\varkappa}^{\beta_{x}} \mid \prod_{x=1}^{k} g_{\varkappa}^{r_{x}}
$$

$$
Q=\prod_{x=1}^{k} q_{\varkappa}^{\prime r_{\varkappa}} \prod_{x=1}^{k} g_{\varkappa}^{r_{\varkappa}}\left(q_{k}^{\prime \prime} f_{k}\right)^{\left[k+\frac{r_{k} \beta}{1-\delta} k\left(\frac{1}{\longleftarrow}+\varepsilon\right)\right]}
$$

den gesuchten Faktor, für den

$$
\left|Q R_{(\varrho)}((\zeta))\right| \geqq 1
$$

5. Es soll nun $\left|Q R_{(\varrho)}((\zeta))\right|$ nach oben abgeschätzt werden, und zwar zunächst $Q$. Für hinreichend großes $q_{\varkappa}=q_{\varkappa}\left(u_{i}, d_{\varkappa}\right)$ gilt auf Grund von (1) und (14)

$$
\prod_{\varkappa=1}^{k} q_{\varkappa}^{\prime r_{\varkappa}} \leqq \prod_{\varkappa=1}^{k} q_{\varkappa}^{\left.\prime \omega_{1}+\delta\right) r_{\varkappa}} \leqq q_{k}^{k r_{k}\left(\omega_{1}+\delta\right)}
$$


Auf Grund von Hilfssatz \pm ist

$$
\prod_{\varkappa=1}^{k} g_{*}^{r_{*}} \leqq q_{k}^{s k r_{k}}
$$

Schließlich gilt wegen der 2. und 3. Voraussetzung des Satzes 1 für $\frac{1}{r_{k}}<\delta$

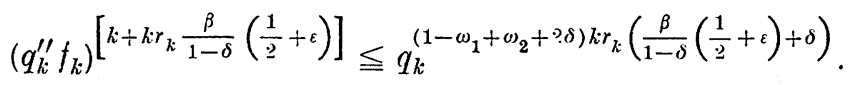

Dann ist insgesamt

mit

$$
|Q| \leqq q_{k}^{k: r_{k} \psi}
$$

$$
\psi=\omega_{1}+2 \delta+\left(1-\omega_{1}+\omega_{2}+2 \delta\right)\left(\frac{\beta}{1-\delta}\left(\frac{1}{2}+\varepsilon\right)+\delta\right) .
$$

Die Abschätzung von $R_{(\varrho)}((\zeta))$ nach oben, die auf Grund von Hilfssatz 2, der Voraussetzung, daß die $\frac{p_{i}}{q_{i}}$ Lösungen von $\left|\alpha-\frac{p_{i}}{q_{i}}\right|<q_{i}^{-\mu}$ sind, und (18) erfolgt, übernehmen wir aus dem Beweis des anfangs erwähnten Satzes ([4], Formel (18)). Sie lautet

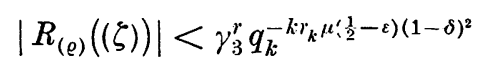

mit $r=\max \left(r_{1}, \ldots, r_{k}\right)$ und einer geeigneten Konstante $\gamma_{3}$. Damit ist

$$
\left|Q R_{(\varrho)}((\zeta))\right| \leqq \gamma_{3}^{r} q_{k}^{k \cdot r_{k} \chi}
$$

mit

$\chi=-\mu\left(\frac{1}{2}-\varepsilon\right)(1-\delta)^{2}+\omega_{1}+2 \delta+\left(1-\omega_{1}+\omega_{2}+2 \delta\right)\left(\frac{\beta}{1-\delta}\left(\frac{1}{2}+\varepsilon\right)+\delta\right)$, wobei $\beta$ durch (15) gegeben ist.

6. Es kann nun gezeigt werden, daß für

$$
\mu=1+\omega_{1}+\omega_{2}\left(\left(1-\omega_{1}\right)\left(2+\omega_{2}\right)+\omega_{1}^{2}\right)+v
$$

mit $v>0$ und hinreichend kleine $\delta, \varepsilon>0$ der Exponent $\chi$ negativ wird.

Da zwischen $\varepsilon$ und $\delta$ bisher keine Beziehung hergestellt wurde, kann $\varepsilon=\delta$ gesetzt werden. Dann gelten für hinreichend kleines $\delta$ folgende Ungleichungen

$$
\left(\begin{array}{l}
1 \\
2
\end{array}-\varepsilon\right)(1-\delta)^{2}>\frac{1}{2}-\delta, \quad \frac{1}{1-\delta}\left(\frac{1}{2}+\varepsilon\right) \leqq \frac{1}{2}+2 \delta .
$$

Aus $\omega_{2} \geqq 1$ folgt $\mu>2$; weil dafür die Behauptung des Satzes bekannt ist, können wir, um die folgende Abschätzung zu vereinfachen, $\omega_{2}<1, \mu \leqq 2$ und damit auch $1-\omega_{1}+\omega_{2}<2$ und $1+\omega_{2}\left(1-\omega_{1}\right)<2$ voraussetzen. Sei ferner $d=\frac{1-\omega_{1}}{\delta}$; wegen $1-\omega_{1}>0$ ist dann $d \neq 0$, und es gilt

$$
\beta \leqq\left(1+\frac{3}{d}\right)\left(1+\omega_{2}\left(1-\omega_{1}\right)+3 \delta\right),
$$

also für hinreichend kleines $\delta$

$$
\begin{aligned}
& \left(1-\omega_{1}+\omega_{2}+2 \delta\right)\left(\frac{\beta}{1-\delta}\left(\frac{1}{2}+\varepsilon\right)+\delta\right) \\
& \quad \leqq\left(1-\omega_{1}+\omega_{2}+2 \delta\right)\left(\left(1+\frac{3}{d}\right)\left(1+\omega_{2}\left(1-\omega_{1}\right)+3 \delta\right)\left(\frac{1}{2}+2 \delta\right)+\delta\right) \\
& \quad \leqq \frac{1}{2}\left(1-\omega_{1}+\omega_{2}\right)\left(1+\omega_{2}\left(1-\omega_{1}\right)\right)+\frac{10}{d}+16 \delta .
\end{aligned}
$$


Damit wird

$\% \leqq-\frac{\mu}{2}+\omega_{1}+\frac{1}{2}\left(1-\omega_{1}+\omega_{2}\right)\left(1+\omega_{2}\left(1-\omega_{1}\right)\right)+\frac{10}{d}+20 \delta \leqq-\frac{v}{2}+\frac{50}{d}$.

Es ist also $\%<0$ für $d>\frac{100}{v}$ oder $\delta<\frac{\left(1-\omega_{1}\right) v}{100}$.

Es sei nun $\delta$ in dieser Weise gewählt. $k$ und $r_{k}$ sind durch die Hilfssätze und im Beweis nur untere Schranken auferlegt worden, die durch geeignete Wahl erfüllt werden können. Dann wird wegen $\chi<0$ die rechte Seite von (20) für hinreichend großes $q_{k}=q_{k}\left(u_{i}, d_{k}\right)$ kleiner als 1 , im Widerspruch zu (19). Damit ist auch die Annahme, der Satz sei falsch, zum Widerspruch geführt.

\section{Beweis von Satz 2}

Den Beweis von Satz 2 beginnen wir mit dem folgenden

Hilfssatz 5. Für die natürtichen Zahlen $u_{v-1}, u_{v}$ sei $\frac{u_{v}}{u_{v-1}} \geqq d>1$. Ist $e_{v}$ der größte Exponent, mit dem die Primzahl $p$ in $u_{\nu}$ ! aufgeht, und $e_{\nu}^{\prime}$ der größte Exponent, mit dem $p$ in $\frac{u_{\nu} !}{u_{\nu-1} !}$ aufgeht, so ist

also für $e_{\nu}^{\prime}>0$

$$
e_{\nu} \leqq \frac{2 d}{d-1}\left(e_{\nu}^{\prime}+1\right)
$$

$$
e_{v} \leqq \frac{4 d}{d-1} e_{v}^{\prime}
$$

Beweis. Aus der bekannten Beziehung

folgt einerseits

$$
e_{v}=\left[\frac{u_{v}}{p}\right]+\left[\frac{u_{\nu}}{p^{2}}\right]+\cdots
$$

$$
e_{v} \leqq \frac{u_{v}}{p}-1 .
$$

Andrerseits gilt wegen $\frac{u_{v}}{u_{v-1}} \geqq d>1$

$$
e_{v}^{\prime} \geqq\left[\frac{u_{v}}{p}\right]-\left[\frac{u_{v}}{d p}\right] \geqq \frac{u_{v}}{p}\left(1-\frac{1}{d}\right)-1 .
$$

Beide Beziehungen liefern zusammen

$$
e_{\nu}^{\prime} \geqq e_{v} \frac{p-1}{p}\left(1-\frac{1}{d}\right)-1 \geqq \frac{e_{v}}{2}\left(1-\frac{1}{d}\right)-1,
$$

also die Behauptung.

Der Beweis von Satz 2 wird wieder indirekt geführt. Unter der Annahme, daß $\xi$ algebraisch sei, können wir die Voraussetzungen von Satz 1 als erfüllt nachweisen, jedoch zeigen, daß dessen Behauptung nicht gilt.

Zunächst folgt unmittelbar aus den Voraussetzungen von Satz 2, daß die angegebene Reihe konvergiert. Ferner kann in der 2. Voraussetzung wegen $c>1$ und $c>d$ auch $d>1$ vorausgesetzt werden. Dann gilt für fast alle $v$

$$
\frac{u_{\nu+1}}{u_{\nu}} \geqq d>1 \text {, }
$$

so daß dies ohne Einschränkung der Allgemeinheit sogleich für alle derartigen Quotienten der gegebenen Reihe vorausgesetzt werden kann. 
Die Folge der in Satz I vorkommenden Näherungswerte $\frac{p_{i}}{q_{i}}=\zeta_{i}$ setzen wir jetzt gleich mit der Folge der Partialsummen $\sum_{\nu=0}^{x} \varrho_{\nu}$, die zu $\varrho_{r} \neq 0$ gehören.

Wir bezeichnen:

$$
\frac{p_{i}}{q_{i}}=\zeta_{i}=\sum_{\nu=0}^{x_{i}} \frac{r_{\nu}}{u_{\nu} !}
$$

und es sei

$$
q_{i}^{\prime} \prod_{y^{\prime}=0}^{\varkappa_{i}} t_{\nu}, \quad q_{i}^{\prime \prime} \mid u_{\varkappa_{i}} !
$$

Es soll zunächst gezeigt werden, daß $\omega_{2}=0$ ist, wozu die Faktoren $f_{i}$ mit $q_{i}^{\prime \prime} f_{i}=u_{\varkappa_{i}}$ ! abzuschätzen sind. Im allgemeinen gilt $q_{\varkappa_{i}} \mid \prod_{\gamma^{\prime}=0}^{\varkappa_{i}-1} t_{v^{\prime}} u_{\varkappa_{i}-1}$ !, und wir nehmen zunächst an, es sei $q_{i-1}=\prod_{\varkappa_{i}}^{\varkappa_{i}} t_{v} u_{\varkappa_{i}-1}$ !. Dann ist mit der Bezeichnung $\prod_{\nu=0}^{\varkappa_{i}} t_{v}=T_{\varkappa_{i}}$

$$
\frac{p_{i}}{q_{i}}=\frac{p_{i-1}}{q_{i-1}}+\frac{s_{\varkappa_{i}}}{t_{\varkappa_{i}} u_{x_{i}} !}=\frac{p_{i-1} t_{x_{i}} u_{\varkappa_{i}}^{\prime}+T_{{\varkappa_{i}-1}_{i}} s_{\varkappa_{i}}}{T_{\varkappa_{i}} u_{\varkappa_{i}-1} ! u_{\varkappa_{i}}^{\prime}}
$$

wobei also $u_{\varkappa_{i}}^{\prime}=\frac{u_{x_{i}} !}{u_{x_{i}-1} !}$ ist. Zunächst denken wir diesen Bruch durch $\left(u_{\varkappa_{i}}^{\prime}, T_{\varkappa_{i}-1} s_{\varkappa_{i}}\right)=v_{\varkappa_{i}}$ gekürzt und kennzeichnen die gekürzten Größen durch einen Querstrich:

$$
\frac{p_{i}}{q_{i}}=\frac{p_{i-1} t_{x_{i}} \overline{u_{x_{i}}^{\prime}}+\overline{T_{x_{i}-1}} \overline{s_{x_{i}}}}{T_{x_{i}} u_{x_{i}-1} ! \overline{u_{x_{i}}^{\prime}}} .
$$

Dann kann ein Primfaktor des Nenners, der in $\overline{u_{x_{i}}^{\prime}}$ enthalten ist, nicht im Zähler aufgehen. Als gemeinsame Faktoren von Zähler und Nenner kommen also nur die höchsten Primzahlpotenzen des Nenners in Frage von Primzahlen, die $\overline{u_{\varkappa_{i}}^{\prime}}$ nicht teilen. Diese sollen jetzt bestimmt werden, und zwar die höchsten derartigen Primzahlpotenzen von $u_{\varkappa_{i}-1}$ !. Offenbar gilt für diese Primzahlen zunächst $p \leqq u_{x_{i}-1}$, und ihre Anzahl $\sigma_{\varkappa_{i}-1}^{\prime}$ kann mit Hilfe des Primzahlsatzes abgeschätzt werden:

$$
\left.\sigma_{\varkappa_{i}-1}^{\prime} \leqq(1+\dot{\delta}) \frac{u_{\varkappa_{i}-1}}{\log u_{x_{i}-1}} \leqq \frac{u_{x_{i}}}{\log u_{x_{i}-1}} \leqq \frac{u_{x_{i}}}{\left(\varkappa_{i}-1\right) \log d}=\sigma_{\varkappa_{i}} \quad \text { für } 1+\delta<d^{3}\right) .
$$

Nimmt man zunächst an, daß $\overline{u_{\varkappa_{i}}^{\prime}}=u_{\varkappa_{i}}^{\prime}$ ist, so gilt für die Primfaktoren von $u_{\varkappa_{i}-1}$ !, die in $u_{\varkappa_{i}}^{\prime}$ nicht aufgehen, mit der Bezeichnung von Hilfssatz $5 e_{\varkappa_{i}}^{\prime}=0$, also $e_{\varkappa_{i}} \leqq \frac{2 d}{d-1}$, und folglich geht eine solche Primzahl $p$ in $u_{\varkappa_{i}-1}$ ! auch nur mit einer Potenz $\leqq \frac{2 d}{d-1}$ auf. Für das größte Potenzprodukt $f_{\varkappa_{i}}^{\prime}$ aller dieser Prim. faktoren von $u_{\varkappa_{i}-1}$ ! besteht daher die Abschätzung

$$
f_{\varkappa_{i}}^{\prime} \leqq u_{\varkappa_{i}}^{v_{x_{i}} \frac{2 d}{d-1}}
$$

${ }^{3}$ ) Diese und alle folgenden Abschätzungen gelten stets nur für hinreichend großes $i$ bzw. $x_{i}$ bzw. $v$, ohne daß dies jeweils erwähnt wird. 
Damit haben wir bisher gezeigt: Ist $\overline{u_{\varkappa_{i}}^{\prime}}=u_{\varkappa_{i}}^{\prime}$, so kann in (22) der Zähler und im Nenner der Ausdruck $u_{\varkappa_{i}}$ ! nur durch den Faktor $f_{\varkappa_{i}}^{\prime}$ gekürzt werden.

Nun ist $u_{\varkappa_{i}}^{\prime}=\overline{u_{\varkappa_{i}}^{\prime}} v_{\varkappa_{i}}$, und $\overline{u_{\varkappa_{i}}^{\prime}}$ kann weniger Primteiler enthalten als $u_{\varkappa_{i}}^{\prime}$, falls $v_{\varkappa_{i}}$ größte Primzahlpotenzprodukte von $u_{\varkappa_{i}}^{\prime}$ enthält. Dann kann der Zähler durch weitere Primzahlpotenzprodukte von $u_{\varkappa_{i}}$ ! teilbar werden. Das Produkte $f_{\varkappa_{i}}^{\prime \prime}$ aller dieser Primzahlpotenzprodukte kann jedoch auf Grund von Hilfssatz 5 abgeschätzt werden:

$$
f_{\varkappa_{i}}^{\prime \prime} \leqq v_{\varkappa_{i}}^{\frac{4 d}{d-1}}
$$

Insgesamt haben wir damit

$$
f_{\varkappa_{i}}=f_{\varkappa_{i}}^{\prime} f_{\varkappa_{i}}^{\prime \prime} \leqq u_{\varkappa_{i}}^{\sigma_{\varkappa_{i}} \frac{2 d l}{d-1}} v_{\varkappa_{i}}^{\frac{4 d}{d-1}}
$$

Dabei hatten wir die Voraussetzung $q_{i-1}=\prod_{y=0}^{\kappa_{i}-1} t_{\nu} u_{\varkappa_{i}-1}$ ! gemacht. Im Falle, daß $q_{i-1}$ nur ein Teiler dieses Produkts ist, ist diese Abschätzung offenbar ebenfalls gültig, so daß (23) in jedem Falle gilt.

Es ist nun die rechte Seite von (23) weiter zu majorisieren. Da $v_{\varkappa_{i}}$ Teiler von $\because-1$

$\prod_{v=11} t_{\nu}\left|s_{\varkappa_{i}}\right|$ ist und wegen $\left|s_{\nu}\right|, t_{\nu}<B^{d^{\nu}}$ gilt

$$
v_{\varkappa_{i}} \leqq \prod_{\nu=0}^{\varkappa_{i}-1} t_{\nu}\left|s_{\varkappa_{i}}\right| \leqq B^{\sum^{\varkappa_{i}} d^{\nu}}=B^{\frac{d^{\varkappa_{i}+1}-1}{l-1}},
$$

also

$$
f_{i} \leqq e^{\frac{2 d}{d-1}} \frac{u_{x_{i}} \log u_{x_{i}}}{\left(x_{i}-1\right) \operatorname{lo} \leq d}+\frac{d^{x_{i}}+1}{d-1} \log \leq 1
$$

Folglich ist

$$
\omega_{2}=\lim _{i \rightarrow \infty} \frac{\log f_{i}}{\log q_{i}} \leqq \lim _{i \rightarrow \infty} \frac{\log f_{i}}{(1-\delta) u_{x_{i}} \log u_{\varkappa_{i}}-\log f_{i}}=0
$$

Aus (24) folgt ebenso, daß $\omega_{1}=0$, und wegen $\frac{u_{\varkappa_{i}}}{u_{\varkappa_{i}-1}} \geqq d>1$ erhält man schließ. lich für $\delta<\frac{d-1}{d+1}$

$$
q_{i-1}<u_{\varkappa_{\varkappa_{i}-1}}^{u_{\varkappa_{i}-1}(1+\delta)}<u_{\varkappa_{i}}^{u_{\varkappa_{i}(1-\delta)}}<q_{i}
$$

Um unter der Annahme, daß $\xi$ algebraisch ist, auch die 4. Voraussetzung des Satzes als erfüllt nachzuweisen, schätzen wir $\left|\xi-\frac{p_{i}}{q_{i}}\right|$ ab. Es ist wegen $\frac{u_{v+1}}{u_{v}} \geqq d$

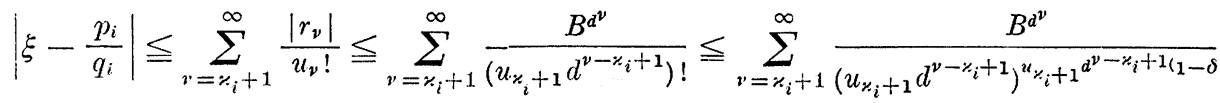

$$
\begin{aligned}
& \leqq \frac{1}{u_{\varkappa_{i}+1}^{u_{\varkappa_{i}+1}}} \sum_{v=\varkappa_{i}+1}^{\infty} e^{l^{\nu}\left(\log B-\left(\nu-\varkappa_{i}+1\right) u_{0} \log d \cdot(1-\delta)\right)} \leqq \frac{\gamma^{d^{*_{i}+1}+1}}{u_{\varkappa_{i}+1}^{\left.u_{\varkappa_{i}+1}+1-\delta\right)}},
\end{aligned}
$$


wobei die letzte Ungleichung gilt, weil der Klammerausdruck im Exponenten nur für endlich viele $v$, unabhängig von $\varkappa_{i}$, positiv ist. $\gamma$ ist dabei eine geeignete Konstante, die nicht von $\varkappa_{i}$ abhängt. Dieser Ausdruck kann weiter abgeschätzt werden; er ist für hinreichend großes $\varkappa_{i}$ kleiner oder gleich

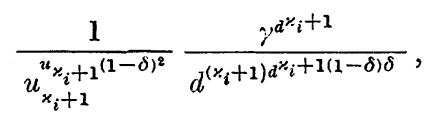

also insgesamt

$$
\left|\xi-\frac{p_{i}}{q_{i}}\right| \leqq \frac{1}{u_{x_{i}}^{u_{\varkappa_{i}} d(1-\delta)^{2}}} .
$$

Wählt man nun $1<\mu<d$ und $0<\delta \leqq \frac{d-\mu}{2(d+\mu)}$, so ist $d(1-\delta)^{2}>\mu(1+\delta)$, also

$$
\frac{1}{u_{x_{i}}^{u_{x_{i}} d(1-\delta)^{2}}} \leqq \frac{1}{u_{\varkappa_{i}}^{u_{x_{i}} \mu(1+\delta)}} \leqq \frac{1}{\left(u_{x_{i}} !\right)^{\mu}}
$$

und damit die 4. Voraussetzung von Satz 1 erfüllt.

Es bleibt nun noch zu zeigen, daß die Behauptung von Satz 1 nicht zutrifft. Um Satz 1 mit $\lim _{i \rightarrow \infty} b_{i}=\infty$ anwenden zu können, muß gezeigt werden, daß $\eta>1$ ist. Wegen $\omega_{1}=\omega_{2}=0$ gilt mit $\delta>0$

$$
\frac{\log q_{i+1}}{\log q_{i}} \geqq \frac{q_{i} d \log \left(q_{i} d\right)(1-\delta)}{q_{i} \log q_{i}(1+\delta)} \geqq d \frac{1-\delta}{1+\delta},
$$

also

$$
\eta=\lim _{i \rightarrow \infty} \frac{\log q_{i+1}}{\log q_{i}} \geqq d>1
$$

Wegen $\lim _{i \rightarrow \infty} b_{i}=\infty$ müßte für die Partialsummen, die mit den in (7) gegebenen Reihengliedern enden, (6) gelten. Offenbar steht das im Widerspruch zur Voraussetzung $3 \mathrm{~b}$ von Satz 2 . Damit ist $\xi$ als transzendent erkannt.

\section{Literatur}

[1] K. Mafler, Ein Analogon zu einem Schneiderschen Satz. Proc. Akad. Wet. Amsterdam 39, $653-640,729-737$ (1936).

[2] —- Arithmetische Eigenschaften einer Klasse von Dezimalbrüchen. Proc. Akad. Wet. Amsterdam 40, 421-428 (1937).

[3] Th. Schnerder, Über die Approximation algebraischer Zahlen. J. reine angew. Math. 175, 182-192 (1936).

[4] —, Zur Annäherung der algebraischen Zahlen durch rationale. J. reine angew. Math. 188, 115-128 (1950).

[5] C. I. Sr EgEL, Ưber Näherungswerte algebraischer Zahlen. Math. Ann. 84, 80-99 (1921). 